\title{
Differential baseline expression and angiotensin II-stimulation of leukemia-associated RhoGEF in vascular smooth muscle cells of spontaneously
} hypertensive rats

\author{
This article was published in the following Dove Press journal: \\ International Journal of Nanomedicine \\ 3 December 2012 \\ Number of times this article has been viewed
}

\author{
Wei-Chiao Chiu' \\ Jyh-Ming Juang ${ }^{1,2}$ \\ Shen-Nan Chang ${ }^{2}$ \\ Cho-Kai Wu² \\ Chia-Ti Tsai \\ Chuen-Den Tseng ${ }^{2}$ \\ Yung-Zu Tseng ${ }^{1,2}$ \\ Ming-Jai Su ${ }^{3}$ \\ Fu-Tien Chiang ${ }^{1,2,4}$ \\ 'Graduate Institute of Physiology, \\ National Taiwan University College \\ of Medicine, ${ }^{2}$ Division of Cardiology, \\ Department of Internal Medicine, \\ National Taiwan University Hospital \\ and National Taiwan University \\ College of Medicine, ${ }^{3}$ Graduate \\ Institute of Pharmacology, National \\ Taiwan University College of \\ Medicine, ${ }^{4}$ Department of Laboratory \\ Medicine, National Taiwan University \\ College of Medicine, Taipei, \\ Taiwan, Republic of China
}

Purpose: Studies to explore angiotensin II (Ang II) and its downstream signaling pathways via Rho guanine nucleotide exchange factors (RhoGEFs) and RhoA signaling are crucial to understanding the mechanisms of smooth muscle contraction leading to hypertension. This study aimed to investigate the Ang II-induced expression of RhoGEFs in vascular smooth muscle cells (VSMCs) of spontaneously hypertensive rats (SHRs) and to identify the possible regulator associated with hypertension.

Methods: Cultured VSMCs of the aorta from SHRs and Wistar-Kyoto (WKY) rats were treated with or without Ang II or Ang II plus Ang II type 2 receptor antagonists. The expression levels of RhoGEF messenger RNA (mRNA) and protein were determined. To evaluate the changes of aortic ring contractile force in response to Ang II, a nonviral carrier system was adopted to deliver the leukemia-associated RhoGEF (LARG) small interfering RNA via nanoparticles into aortic rings.

Results: The baseline mRNA levels of three RhoGEFs in cultured VSMCs of WKY rats did not increase with age, but they were significantly higher in 12-week-old SHRs than in 5-week-old SHRs. Expression levels of LARG mRNA were higher in SHRs than in age-matched WKY rats. The baseline LAGR protein of 12-week-old SHRs was about four times higher than that of WKY rats of the same age. After Ang II-stimulation, LAGR protein expression was significantly increased in 12-week-old WKY rats but remained unchanged in 12-week-old SHRs. LARG small interfering RNA was successfully delivered into aortic rings using nanoparticles. LARG knockdown resulted in 12-week-old SHRs showing the greatest reduction in aortic ring contraction.

Conclusion: There were differences in age-related RhoGEF expression at baseline and in response to Ang II-stimulation between SHRs and WKY rats in this study. Nanotechnology can assist in studying the silencing of LARG in tissue culture. The findings of this study indicate that LARG gene expression may be associated with the genesis of hypertension in SHRs.

Keywords: Rho guanine nucleotide exchange factor, leukemia-associated Rho guanine nucleotide exchange factor, Wistar-Kyoto rats, nanoparticle delivery, hypertension

\section{Introduction}

Hypertension is a worldwide problem contributing to major cardiovascular morbidity and mortality. The pathogenesis of hypertension is associated with physiological and biochemical changes in vascular walls. These changes include excessive contractions and hypertrophy of vascular smooth muscle cells (VSMCs) that lead to an increase in peripheral resistance. ${ }^{1}$ Although increased vascular resistance is known to be involved 
in the pathogenesis of hypertension, the underlying molecular mechanisms are not well understood. ${ }^{2}$

The extent of VSMC contraction depends on the phosphorylated level of myosin light chain (MLC) that is regulated by the balance between the activity of MLC kinase and MLC phosphatase (MLCP). The mediated regulation of vascular tone through MLCP and upstream molecules (monomeric G protein RhoA/Rho kinase) is known as calcium $\left(\mathrm{Ca}^{2+}\right)$ sensitization. ${ }^{3-5}$ As well as acting directly on vascular smooth muscle as a potent vasoconstrictor in a $\mathrm{Ca}^{2+}$-dependent manner, Ang II also activates the RhoA/Rho kinase signaling transduction pathway and increases $\mathrm{Ca}^{2+}$ sensitization of VSMCs through Rho guanine nucleotide exchange factors (RhoGEFs). ${ }^{1,6-8}$ The RhoGEFs are a large family of structurally related proteins comprising $\sim 70$ members in the human genome. ${ }^{9}$ Among them, the small subfamily of regulators of G-protein signaling domaincontaining RhoGEFs - including p115-RhoGEF, PSD-95/ Disc-large/ZO-1 homology (PDZ)-RhoGEF, and leukemiaassociated RhoGEF (LARG) - is the only one subject to regulation by $\mathrm{G} \alpha 12 / 13$. These regulators of G-protein signaling domain-containing RhoGEFs mediate the activation of RhoA protein by exchanging guanosine diphosphate for guanosine triphosphate. ${ }^{9,10}$ Misregulated G12/13 signaling via RhoGEFs is involved in multiple pathophysiological conditions such as cancer, cardiovascular diseases, and hypertension; therefore, specific targeting of the related signaling pathway provides novel therapeutic approaches. ${ }^{11}$

Guilluy et $\mathrm{al}^{1}$ have suggested that both the increases of intracellular $\mathrm{Ca}^{2+}$ concentration and $\mathrm{Ca}^{2+}$ sensitization of myofilament are necessary for Ang II-induced vasoconstriction. The authors found the increase of intracellular $\mathrm{Ca}^{2+}$ concentration induced by Ang II was not sufficient to cause sustained vasoconstriction and therefore a RhoA-dependent $\mathrm{Ca}^{2+}$-sensitivity mechanism is required to allow persistent contractions. ${ }^{1}$ Thus, if the $\mathrm{Ca}^{2+}$-sensitization pathway develops a continuous activation that could cause a prolonged contraction of VSMCs in the arteries, the spontaneous tone may increase, leading to elevated peripheral resistance and consequent hypertension.

The spontaneously hypertensive rat (SHR) strain has been extensively used as a model for hypertension research, although the etiology of hypertension genesis in SHRs is not completely understood. Some previous studies have revealed possible mechanisms, including excessive vasoreactivity, overactivation of the sympathetic nervous system, salt sensitivity, and so forth. ${ }^{12,13}$ It has also been reported that high angiotensin converting enzyme activity and high angiotensin II (Ang II) receptor density existed in the vascular wall of young SHRs, causing medial thickening, hypertrophy, and proliferation as well as vasoconstriction in the adult SHR. ${ }^{14}$

The Ang II/RhoGEF/RhoA/MLCP pathway has been investigated in SHRs and WKY rats. No significant differences in expression levels among the tested RhoGEFs (p115-RhoGEF, PDZ-RhoGEF, and LARG) were found between 4-week-old SHRs and WKY rats of the same age, but the messenger RNA (mRNA) level in 12-week-old SHRs was observed to be higher than that in 12-week-old WKY rats. ${ }^{15}$ Furthermore, Moriki et $\mathrm{al}^{2}$ reported no difference in protein levels of RhoA signaling pathway-related molecules - including RhoA, Rho kinase, myosin phosphatase target subunit 1 (MYPT1), CPI-17 (protein kinase C downstream target, a protein kinase $\mathrm{C}$-potentiated inhibitor protein of $17 \mathrm{kDa}$ ), and MLC kinase - in the VSMCs of 10- to 14-week-old SHRs and WKY rats, although the authors did report a greater level of RhoA activity and extent of MYPT1 phosphorylation in the SHRs than in the WKY rats. Currently, few studies have reported the effects of Ang II-stimulation on the expressions of various RhoGEFs in SHRs and WKY rats at different ages. Which member of RhoGEFs as a regulator contributes to hypertension genesis in SHRs has not been consistently reported. ${ }^{1,7,15}$

The present authors' previous study has demonstrated that Ang II upregulated only LARG gene expression through Ang II type 1 receptors $\left(\mathrm{AT}_{1}\right)$ in the VSMCs of Sprague Dawley ${ }^{\circledR}$ rats. After inducing an increase in RhoA activity and phosphorylation of MYPT1, LARG enhanced the Ang II-induced increase of $\mathrm{Ca}^{2+}$ sensitivity in VSMCs; in contrast, ex vivo knockdown of LARG suppressed the aortic ring contraction. ${ }^{16}$

The use of small interfering RNA (siRNA) as a powerful tool to perform gene knockdown is now well established. ${ }^{17}$ A major challenge in the application of siRNAs is the intracellular delivery to specific tissues expressing the target gene. siRNA delivery systems are currently divided into viral and nonviral vectors. Although viral vectors usually achieve more efficient transfection, the immunogenicity of the virus also resides within the experimental tissues and cells, which can cause teratogenesis or mutation. Research using nanosized nonviral carriers for siRNA delivery in medical applications has been an exciting emerging research field in recent years. ${ }^{18}$

In the present study, the authors aimed both to investigate the baseline expression of RhoGEF mRNA in SHRs and WKY rats at different ages and to explore the effects 
of Ang II-stimulation through the $\mathrm{AT}_{1}$ receptor on RhoGEF mRNA and protein expression levels. Finally, a gene knockdown experiment was conducted using nonviral carrier systems, nanoparticles, and liposomes to deliver siRNAs into the aortic rings to clarify the potential role of LARG in aortic ring contraction in SHRs and WKY rats.

\section{Materials and methods Animals}

All animal procedures were performed in accordance with protocols approved by the Institutional Animal Care and Use Committee of the National Taiwan University College of Medicine, Taipei, Taiwan, Republic of China (Approval No 20090097). Male SHRs and WKY rats at ages of 5 (body weight range: $150-180 \mathrm{~g}$ ) and 12 weeks (body weight range: 275-300 g) were purchased from the National Laboratory Animal Center, Taipei, Taiwan, Republic of China.

\section{Tissue preparation and cell culture}

VSMCs were prepared from the thoracic aorta of 5- and 12-week-old male SHRs and WKY rats. The blood pressure of each rat was measured to confirm the presentation of hypertension in the SHRs (mean blood pressures for 5- and 12-week-old SHRs: $130 \pm 7.4 \mathrm{mmHg}$ and $192 \pm 7.0 \mathrm{mmHg}$, respectively; mean blood pressures for 5- and 12-weekold WKY rats: $120 \pm 6.2 \mathrm{mmHg}$ and $122 \pm 5.7 \mathrm{mmHg}$, respectively). The rats were then anesthetized with sodium pentobarbital $(50 \mathrm{mg} / \mathrm{kg}$ body weight, administered intraperitoneally). The thoracic aortas were rapidly isolated and immersed in Dulbecco's Modified Eagle's Medium. The samples were then carefully cleaned of connective tissue and adherent fat. The isolated aorta was cut open longitudinally, followed by endothelial removal by gently grazing the intimal surface with sharp scissors. The denuded aortas were cut into approximately $3 \mathrm{~mm}^{2}$ sections and placed, with the intimal side down, in $30 \mathrm{mmol} / \mathrm{L}$ dishes. Medium 199 supplemented with fetal bovine serum (20\%) and antibiotics $(100 \mathrm{U} / \mathrm{mL}$ of penicillin and $100 \mu \mathrm{g} / \mathrm{mL}$ of streptomycin) was gently added to cover the tissues without disturbing the orientation of the explants. These preparations were then cultured at $37^{\circ} \mathrm{C}$ under a humidified atmosphere of $95 \%$ air and 5\% carbon dioxide. VSMCs were allowed to grow out from the explants for 7-10 days after the tissues were removed. After confluence, the cells were harvested using trypsin $(0.05 \%)$ and were then grown in $25 \mathrm{~cm}^{2}$ flasks (first passage). The cultures were passaged twice weekly by harvesting with trypsin and seeding at a $1: 4$ ratio in $75 \mathrm{~cm}^{2}$ flasks. The cells showed a characteristic "hills and valleys" growth pattern of cultured VSMCs and exhibited $>95 \%$ positive immunostaining for smooth muscle $\alpha$-actin. Subcultured passage five VSMC samples were used in the experiments. ${ }^{19-22}$ When the cells were approximately $70 \%$ confluent, the medium was replaced with serum-free medium and cultured for 24 hours. Cells were then stimulated with Ang II $(0.1 \mu \mathrm{mol} / \mathrm{L})$ or Ang II $(0.1 \mu \mathrm{mol} / \mathrm{L})$ plus Ang II type 2 receptor antagonist PD123319 $(1 \mu \mathrm{mol} / \mathrm{L})$ for 30 minutes or 3,6 , or 9 hours.

\section{Isolation of total RNA and reverse transcription}

Total RNA from cultured cells was isolated using TRIzol ${ }^{\mathbb{B}}$ reagent (Life Technologies Corporation, Carlsbad, CA) according to the manufacturer's instructions. All reverse transcription reactions were performed using a High Capacity cDNA Reverse Transcription Kit (Applied Biosystems, Foster City, CA) according to the manufacturer's instructions. The complementary DNA (cDNA) was used as the template for a quantitative real-time polymerase chain reaction (qPCR) assay.

\section{QPCR assay}

The qPCR assay was performed using a 7000 Sequence Detection System (Applied Biosystems), and cDNA generated from total RNA (30 ng) was used for each reaction. The PCR reaction mixture consisted of $2 \times$ concentration of TaqMan ${ }^{\circledR}$ Universal PCR Master Mix (Applied Biosystems); $20 \times$ TaqMan Gene Expression Assay Mix (Applied Biosystems) for rat p115-RhoGEF (assay ID: Rn00572505 m1), PDZ-RhoGEF (ID: Rn00576857_m1), LARG (ID: Rn01417838_m1), or glyceraldehyde-3-phosphate dehydrogenase (GAPDH) (ID: Rn99999916_s1); and cDNA diluted in RNase-free water to a final volume of $20 \mu \mathrm{L}$. Samples were amplified with 40 cycles of $95^{\circ} \mathrm{C}$ for 15 seconds (denaturation) and $60^{\circ} \mathrm{C}$ for 1 minute (annealing and extension). The amount of each target mRNA was normalized to the internal control (GAPDH) and calculated using the comparative cycle threshold $\left(\mathrm{C}_{\mathrm{t}}\right)$ method as follows:

$$
\text { target mRNA } \text { exp }_{\text {/ }} \text { arget } \text { mRNA }_{\text {con }}=2^{-\Delta \Delta \mathrm{C}_{\mathrm{t}}}
$$

where,

$$
\begin{aligned}
\Delta \Delta \mathrm{C}_{\mathrm{t}}=\Delta \mathrm{C}_{\mathrm{t}, \text { exp }}-\Delta \mathrm{C}_{\mathrm{t}, \text { con }}= & \left(\mathrm{C}_{\mathrm{t}, \text { target mRNA }}-\mathrm{C}_{\mathrm{t}, \mathrm{GAPDH}}\right)_{\exp } \\
& -\left(\mathrm{C}_{\mathrm{t}, \text { target mRNA }}-\mathrm{C}_{\mathrm{t}, \mathrm{GAPDH}}\right)_{\text {con. }}
\end{aligned}
$$

$\Delta \mathrm{C}_{\mathrm{t} \text { exp }}$ (the change in $\mathrm{C}_{\mathrm{t}}$ ) is the target mRNA's $\mathrm{C}_{\mathrm{t}}$ value in the experimental group (target gene; treated duration of 30 minutes or 3 , or 6 hours), and $\Delta \mathrm{C}_{\mathrm{t}, \mathrm{con}}$ is the $\mathrm{C}_{\mathrm{t}}$ value of 
target mRNA in the control group (target gene; 0 hours; unstimulated conditions). ${ }^{23}$

\section{Protein extraction and Western blot analysis}

Culture dishes were placed on ice and washed twice with icecold phosphate buffered saline. All subsequent procedures were performed over ice. Lysis buffer (cold phosphate buffered saline solution, $\mathrm{pH} 7.4$, containing $1 \mathrm{mmol} / \mathrm{L}$ ethylenediaminetetraacetic acid (EDTA), $25 \mathrm{mmol} / \mathrm{L}$ 4-(2-hydroxyethyl)-1-piperazineethanesulfonic acid, $150 \mathrm{mmol} / \mathrm{L}$ sodium chloride, $10 \mathrm{mmol} / \mathrm{L}$ magnesium chloride, $10 \%$ glycerol, and 1\% Igepal ${ }^{\circledR}$ CA-630 [Sigma-Aldrich, St Louis, $\mathrm{MO}]$ ) was added to the plates, followed by incubation on ice for 2 minutes. Cells were scraped off, transferred to microcentrifuge tubes, sonicated for 5 seconds, and then centrifuged at $12,000 \times \mathrm{g}$ for 15 minutes at $4^{\circ} \mathrm{C}$. The supernatant was kept on ice and the pellet was discarded. Aortas were snap frozen in liquid nitrogen for aortic tissue extraction. Tissues were homogenized in ice-cold homogenization buffer, containing $100 \mathrm{mmol} / \mathrm{L}$ Tris hydrochloride (pH 7.4), $1 \mathrm{mmol} / \mathrm{L}$ ethylene glycol tetraacetic acid, $1 \mathrm{mmol} / \mathrm{L}$ EDTA, $1 \mathrm{mmol} / \mathrm{L}$ phenylmethylsulfonyl fluoride, and $1 \mathrm{mmol} / \mathrm{L}$ sodium orthovanadate. After the homogenates were centrifuged at $12,000 \times \mathrm{g}$ for 15 minutes at $4^{\circ} \mathrm{C}$, the supernatant was transferred to a fresh tube kept on ice and the pellet was discarded. Protein concentration was determined using a bicinchoninic acid protein assay kit (Thermo Fisher Scientific Inc, Waltham, MA).

Protein $(40 \mu \mathrm{g})$ was separated by electrophoresis on a $12 \%$ or $6 \%$ polyacrylamide gel and then transferred onto polyvinylidene difluoride membrane. Nonspecific binding sites were blocked using Pierce Protein-Free Blocking Buffer (Thermo Fisher Scientific Inc) for 1 hour at $24^{\circ} \mathrm{C}$. Membranes were incubated overnight at $4^{\circ} \mathrm{C}$ with anti-LARG (1:1000), anti-p115-RhoGEF (1:1000), anti-PDZ-RhoGEF (1:1000) (Santa Cruz Biotechnology, Inc, Santa Cruz, CA), or anti-GAPDH (1:1000) (EMD Millipore, Billerica, MA). Following exposure to a horseradish peroxidase-conjugated secondary anti-mouse or anti-rabbit antibody, the membrane was subjected to enhanced chemiluminescence reagent (EMD Millipore Corporation, Billerica, MA). Signals were visualized using a UVP imaging system (UVP, LLC, Upland, $\mathrm{CA}$ ), or with X-ray film, and the results were expressed as the densitometric ratio of RhoGEF to GAPDH.

\section{Tissue preparation and isometric force recording}

The 12-week-old SHRs and WKY rats were anesthetized with sodium pentobarbital $(50 \mathrm{mg} / \mathrm{kg}$ body weight, administered intraperitoneally) and the thoracic aortas were immediately excised. The adventitial tissue was cleaned in physiological saline solution (Krebs-Henseleit solution [KHS], pH 7.4) of the following composition: $118 \mathrm{mmol} / \mathrm{L}$ sodium chloride, $4.7 \mathrm{mmol} / \mathrm{L}$ potassium chloride, $1.2 \mathrm{mmol} / \mathrm{L}$ potassium dihydrogen phosphate, $1.9 \mathrm{mmol} / \mathrm{L}$ calcium chloride dihydrate, $1.2 \mathrm{mmol} / \mathrm{L}$ magnesium sulfate, $25 \mathrm{mmol} / \mathrm{L}$ sodium bicarbonate, $11.7 \mathrm{mmol} / \mathrm{L}$ glucose, and $0.03 \mathrm{mmol} / \mathrm{L}$ EDTA. The aortas were cut into $3 \mu \mathrm{m}$ rings. To eliminate the possible effects of nitric oxide, the endothelium was gently removed by gently rubbing the endothelial surface with the edge of a pair of forceps. Aortic rings were incubated with culture medium containing siRNA. After incubation, the aortic rings were mounted in myograph organ baths containing KHS at $37^{\circ} \mathrm{C}$ and gassed with $95 \%$ oxygen and $5 \%$ carbon dioxide. Isometric force generation was recorded with a force transducer (WPI Fort25, World Precision Instruments Inc, Sarasota, FL) and a recorder (CED micro 1401, Cambridge Electronic Design Ltd, Cambridge, United Kingdom). Rings were stretched to $15 \mathrm{mN}$ tension and equilibrated for 120 minutes. During this procedure, KHS was refreshed every 30 minutes and rings were re-stretched as required. After equilibration, the rings were contracted by phenylephrine $(0.1 \mu \mathrm{mol} / \mathrm{L})$. Acetylcholine $(1 \mu \mathrm{mol} / \mathrm{L})$ was added during the plateau phase of contraction to verify efficient removal of endothelium (no relaxation). The drugs were washed out until the contractile response returned to baseline levels. Thereafter, contractile response curves to Ang II were recorded. ${ }^{24-26}$

\section{siRNA delivery}

A nonviral carrier system with a combination of nanoparticles and liposomes was chosen to deliver the siRNAs into the aortic rings in this study. A lipid-based formulation with the use of siPORT NeoFX transfection agent (catalog number AM4511; Life Technologies Corporation) was used to enhance the transfer of vectors. Aortic rings were incubated with culture medium containing either siRNA directed against LARG $(10 \mathrm{nmol} / \mathrm{L})$ or scrambled siRNA $(10 \mathrm{nmol} / \mathrm{L})$ for 48 hours at $37^{\circ} \mathrm{C} . .^{27,28}$ The siRNAs were introduced by siPORT NeoFX transfection agent according to the manufacturer's instructions. LARG siRNA (catalog number 4390771; Ambion) sequences were as follows: sense, 5'-GCAUCGAUCUGUAUACACUtt-3'; antisense, 5'-AGUGUAUACAGAUCGAUGCtt-3'. Scrambled siRNA, a siRNA sequence not homologous to any known gene, was also purchased from a commercially available resource (cata$\log$ number 4390843; Ambion). After incubation, a portion of the rat aortic rings was snap frozen in liquid nitrogen for 
protein extraction and then subjected to Western blot analysis to verify the knockdown efficiency of LARG. The remaining aortic rings were used to perform experiments on isometric force measurements.

\section{Statistical analysis}

Data are expressed as the mean plus or minus the standard deviation. Results were evaluated using analysis of variance and the unpaired Student's $t$-test. A $P$-value of $<0.05$ was considered significant.

\section{Results}

\section{RhoGEF mRNA expression in SHRs and WKY rats: baseline expression}

The levels of mRNA transcripts examined by qPCR indicated that there was no age-related difference in baseline levels of RhoGEF mRNA between 5-week-old and 12-weekold WKY rats (Figure 1, white bars in WKY rat groups). However, significant age-dependent changes were observed in SHRs $(P<0.01)$, showing that the baseline level of RhoGEF mRNA in 12-week-old SHRs was higher than that in 5-week-old SHRs (Figure 1, white bars in SHR groups). Furthermore, the cultured aortic VSMCs from 12-week-old SHRs expressed a significantly higher level of LARG mRNA than those from WKY rats $(P<0.01$; Figure 1C, 12-week-old WKY rats versus 12 -week-old SHRs, white bars).

\section{Effects of Ang II-stimulation on mRNA expression levels}

As presented in Figure 1A, stimulation with $0.1 \mu \mathrm{mol} / \mathrm{L}$ of Ang II showed no significant effect on the levels of p115RhoGEF mRNA expression at all time intervals studied (30 minutes and 3 and 6 hours) in both rats of two different age groups (Figure 1A). A similar pattern, but lower overall, of PDZ-RhoGEF mRNA expression after Ang II-stimulation was also observed (Figure 1B). However, the levels of LARG mRNA expression were significantly increased in 5- and 12-week-old WKY rats $(P<0.01)$, peaking at 30 minutes after Ang II-stimulation (Figure 1C). In 5-week-old SHRs, there was a slight increase but significantly delayed (at 6 hours) expression of LARG mRNA (Figure 1C); however, the expression levels of LARG mRNA were not changed after Ang II-stimulation in 12-week-old SHRs (Figure 1C).

\section{Protein expression of LARG in SHRs and WKY rats}

Figure $2 \mathrm{~A}$ and $\mathrm{B}$ show a significant increase of $\mathrm{LARG}$ protein in 5- and 12-week-old WKY rats at 6 and 9 hours after
Ang II-stimulation in the presence of PD123319, an Ang II type 2 receptor antagonist. The LARG protein levels were only slightly increased at 9 hours after stimulation in 5-week-old SHRs (Figure 2A). The baseline expression of LARG protein observed in 12-week-old SHRs was significantly greater than that observed in WKY rats of the same age (Figure 2B, white bars, $P<0.01)$. However, LARG protein expression remained unchanged in 12-week-old SHRs after stimulation (Figure 2B). Similar results were obtained in both rats stimulated by $\mathrm{AT}_{1}$ agonist $\mathrm{Val}^{5}$-Ang II (data not shown).

The protein expression levels of p115-RhoGEF and PDZ-RhoGEF were consistent with the corresponding mRNA expression levels, showing no significant change after stimulation with Ang II in the presence of PD123319 (Figure 2).

\section{Effects of LARG siRNA on spontaneous tone and vasoconstriction of the aortic rings in SHRs}

Figure 3A shows the efficiency of LARG protein knockdown in both SHR and WKY rat aortic rings, indicating that the siRNAs were successfully delivered into the aortic rings by using nanoparticle liposomes as a vector. The representative curves of Ang II-induced contraction in endothelium-denuded aortic rings of WKY rats and SHRs are displayed in Figure 3B1 and B2. The level of spontaneous tone developed by the isolated SHR aortic rings was $30.9 \% \pm 5.3 \%$ of phenylephrine-induced maximal contraction (Figure 3B2). The force of contraction from SHR aortic rings pretreated with LARG siRNA was significantly weaker than that from the aortic rings pretreated with scrambled siRNA in response to Ang II $(39.7 \% \pm 3.4 \%$ versus $94.6 \% \pm 0.9 \%$, normalized to phenylephrine-induced maximal contraction, $P<0.01$, in Figure 3B2, B4, and C). Moreover, the SHR aortic rings pretreated with LARG siRNA failed to develop significant spontaneous tone (Figure 3B4). The force of contraction of LARG siRNA pretreated aortic rings from WKY rats was also weaker than that from the aortic rings pretreated with scrambled siRNA in response to Ang II $(40.0 \% \pm 1.8 \%$ versus $79.7 \% \pm 2.0 \%$, normalized to phenylephrine-induced maximal contraction, $P<0.01$, in Figure 3B1, B3, and C). The effects of LARG knockdown were observed in both SHRs and WKY rats, indicating that LARG was essential for Ang II-induced vasoconstriction. It would be most important to evaluate the difference in the degree of impact between SHRs and WKY rats. After pretreatment with LARG siRNA, the reduction of aortic ring force was greater in SHRs than in WKY rats $(58.0 \% \pm 2.6 \%$ versus $49.9 \% \pm 1.3 \%$, normalized to scrambled-siRNA-treated control, $P<0.01$, in Figure 3B3, B4, and C). 

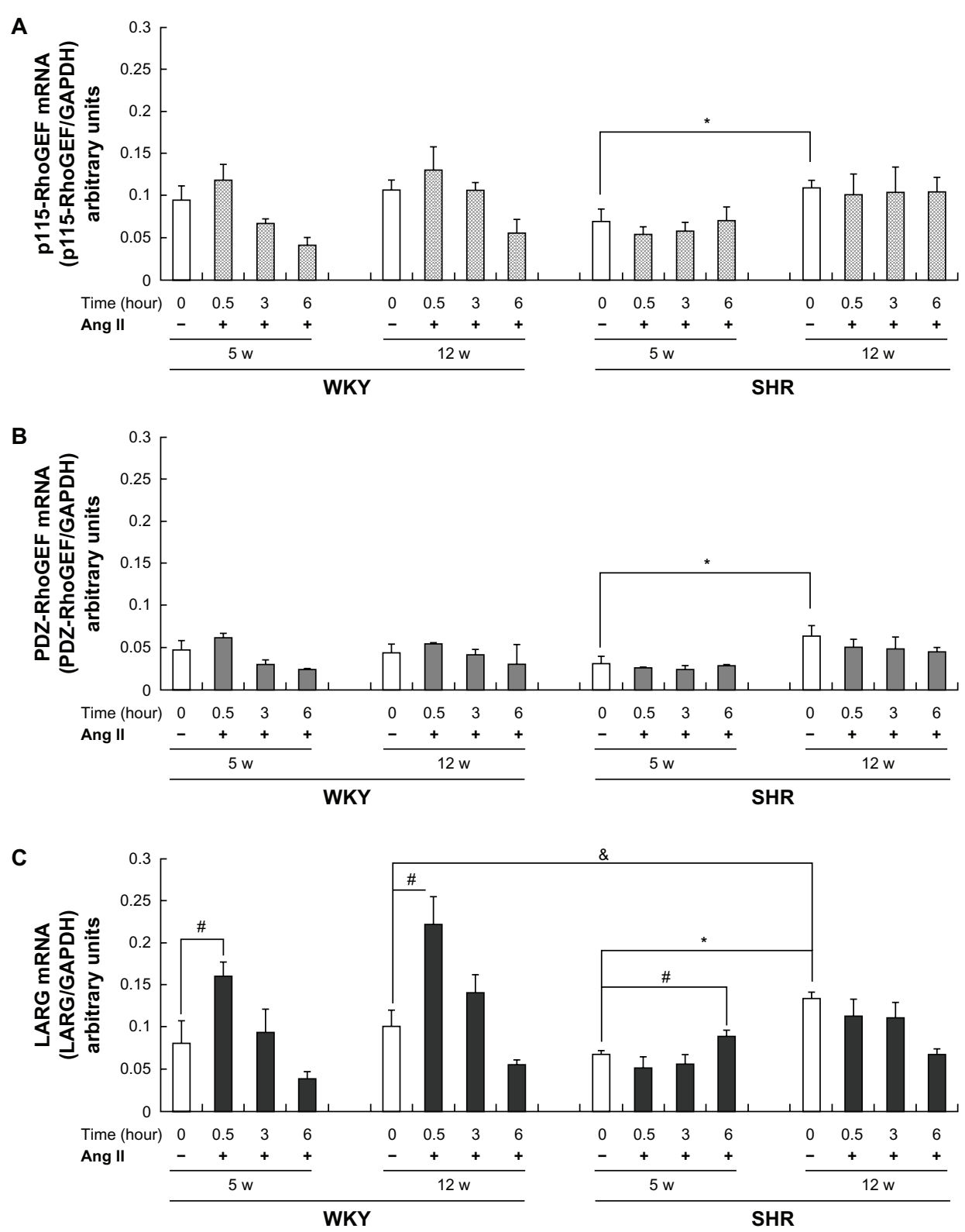

Figure I Rho guanine nucleotide exchange factor (RhoGEF) messenger RNA (mRNA) expression in rat vascular smooth muscle cells (VSMCs) at baseline and after angiotensin II (Ang II) stimulation. VSMCs from 5- and I2-week-old (5W and I2W, respectively) spontaneously hypertensive rats (SHRs) and Wistar-Kyoto (WKY) rats were treated with or without $0.1 \mu \mathrm{mol} / \mathrm{L}$ of Ang II for the indicated times. The mRNAs of (A) PII5-RhoGEF, (B) PSD-95/Disc-large/ZO-I homology (PDZ)-RhoGEF, and (C) leukemia-associated RhoGEF (LARG) were measured by quantitative real-time polymerase chain reaction and were normalized to glyceraldehyde-3-phosphate dehydrogenase (GAPDH). Age-related differences were observed in SHRs but not in WKY rats: the level of LARG expression detected in the I2W group of SHRs was higher than that for the 5W group of SHRs. LARG expression was upregulated by Ang II stimulation in the 5W and I2W groups of WKY rats and in the $5 \mathrm{~W}$ group of SHRs, but no significant change in the $12 \mathrm{~W}$ group of SHRs was observed.

Notes: Data presented as the means of six independent experiments plus or minus standard deviation; $* P<0.0$ I, between rats of the same strain, baseline expression (white bar), I2W groups versus $5 \mathrm{~W}$ groups; ${ }^{\sharp P}<0.0 \mathrm{I}$, within subgroup, Ang II-stimulated (black bar) versus baseline expression (white bar); ${ }^{\&} P<0.0 \mathrm{I}$, baseline expression, I $2 \mathrm{~W}$ group of SHRs versus $12 \mathrm{~W}$ group of WKY rats.

\section{Discussion}

Understanding the regulation of Ang II-mediated activation of RhoGEFs/RhoA signaling is important in many disease conditions, as it represents a possible approach for the treatment of some diseases. The VSMCs of aortas from SHRs have been widely used as a model for investigating the pathogenesis of hypertension. siRNA has become a critical strategy in gene therapy and has been used for functional screening for RhoGEFs associated with VSMCs remodeling. ${ }^{29}$ However, many challenges in using siRNA therapy (such as "off target" effects, immune stimulation, and delivery issues) remain to be overcome. ${ }^{30}$ Appropriate delivery is of utmost importance. Given that the use of nanoparticles to deliver siRNA is an effective approach 
A

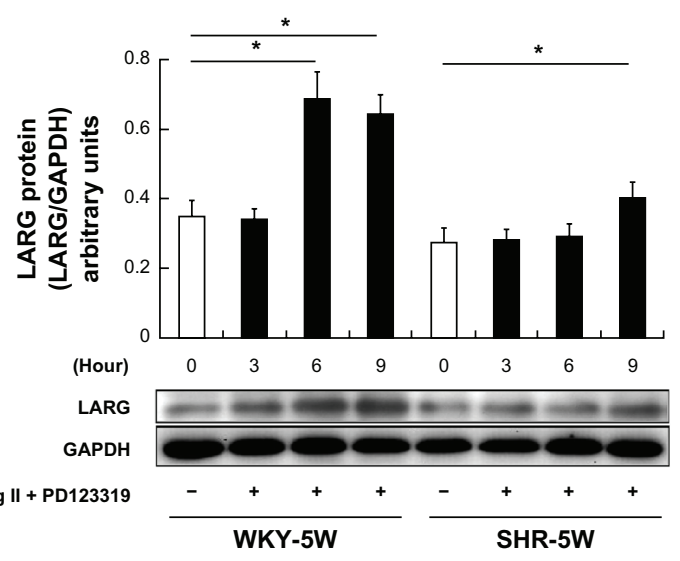

B

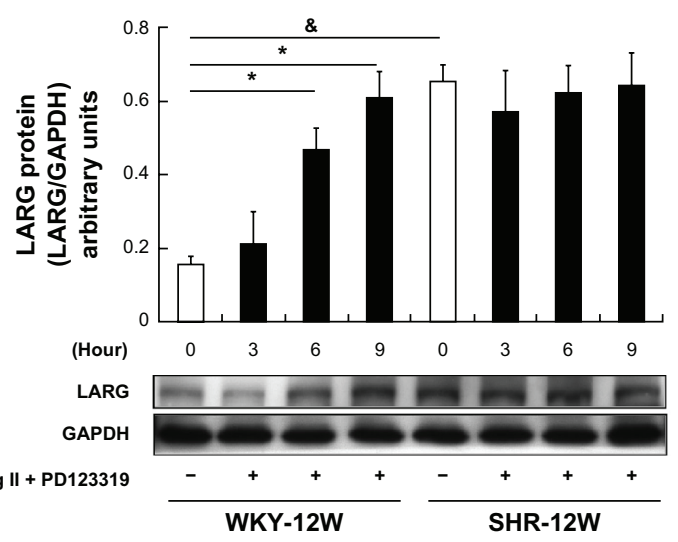

C
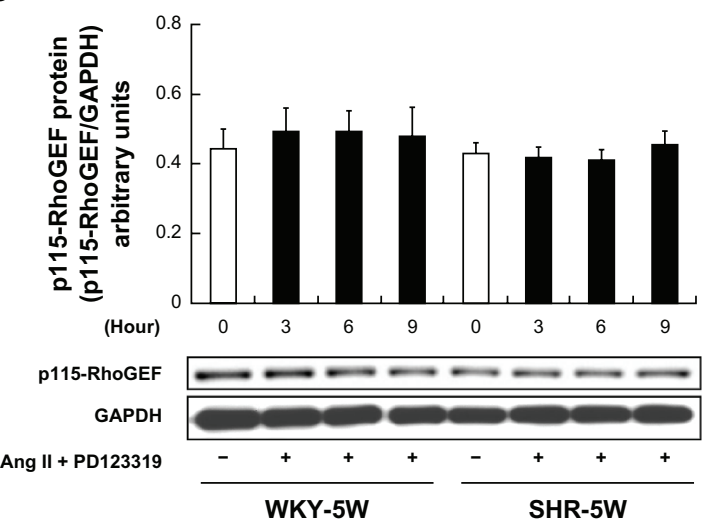

D
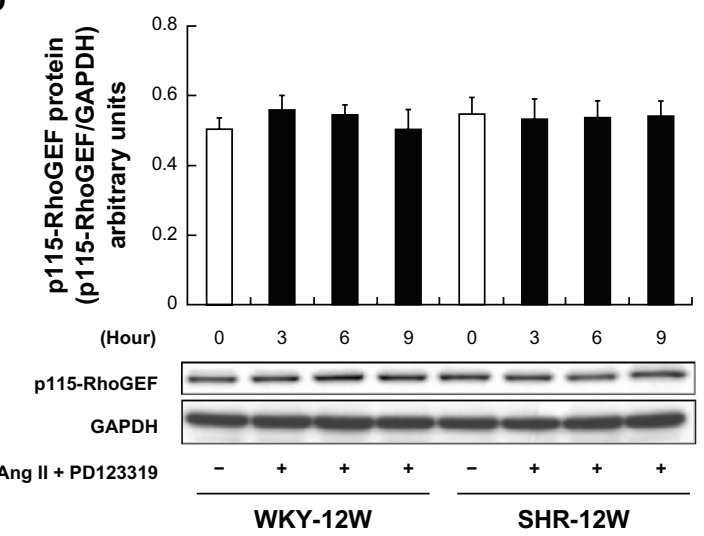

Figure 2 (Continued)
E

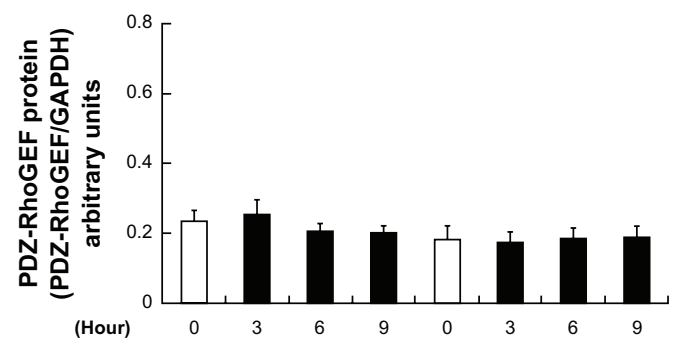

PDZ-RhoGEF

GAPDH

Ang II + PD123319

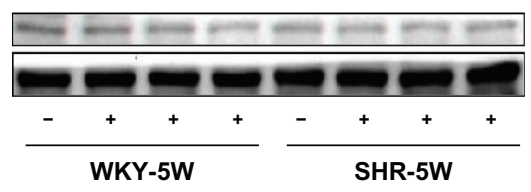

$\mathbf{F}$

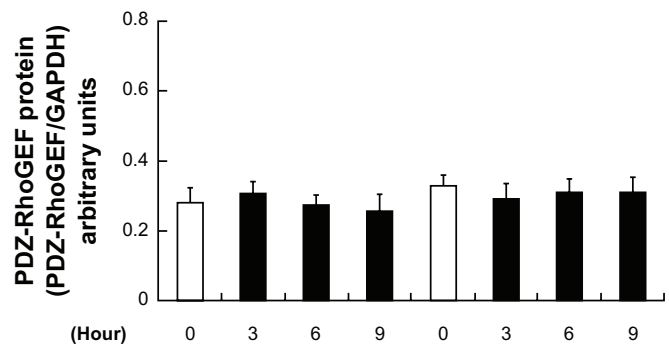

PDZ-RhoGEF

GAPDH

Ang II + PD123319

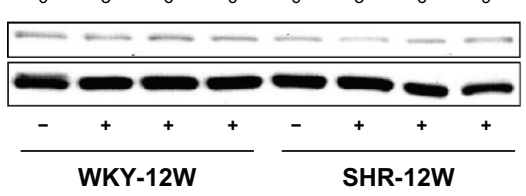

Figure 2 Comparison of Rho guanine nucleotide exchange factor (RhoGEF) protein expression at baseline and after angiotensin II (Ang II) type I receptor activation in rat vascular smooth muscle cells: the protein expression levels of $(\mathbf{A}, \mathbf{B})$ leukemiaassociated RhoGEF (LARG), (C, D) pl I5-RhoGEF, and (E, F) PSD-95/Disc-large/ ZO-I homology (PDZ)-RhoGEF in 5- and 12-week-old Wistar-Kyoto (WKY) rats (WKY-5W and WKY-I2W, respectively) and spontaneously hypertensive rats (SHRs) (SHR-5W and SHR-12W, respectively) at the indicated time points after stimulation by Ang II $(0.1 \mu \mathrm{mol} / \mathrm{L})$ in the presence of Ang II type 2 receptor antagonist PDI233 I 9 (I $\mu \mathrm{mol} / \mathrm{L}$ ) were compared. Western blot analysis was used to measure protein levels, with glyceraldehyde-3-phosphate dehydrogenase (GAPDH) as the internal control. Baseline expression levels of LARG protein were higher in the SHR-I2W group than in the WKY-12W group. LARG protein was regulated in the WKY-I2W group after stimulation, but not in the SHR-12W group.

Notes: Data are presented as the means of six independent experiments plus or minus standard deviation; white bars indicate the baseline expression control; Western blot images are from representative experiments; $* p<0.01$, protein expression levels at the indicated time points versus baseline; ${ }^{\circledR} P<0.01$, baseline expression levels for the WKY-I2W group versus the SHR-I2W group.

to enhance gene delivery to reach the intended target, and considering the main objectives of the study, the authors chose to use a commercially available vector delivery system and nanoparticles (siPORT NeoFX transfection agent).

There were three major findings revealed in this study. First, the baseline expression levels of the three RhoGEF mRNAs from WKY rats were not increased with age; however, the changes observed with age were significant in SHRs. Second, the degree of LARG mRNA upregulation by Ang II-stimulation was greater in WKY rats than in SHRs. The Ang II-induced significant increase in LARG 
A

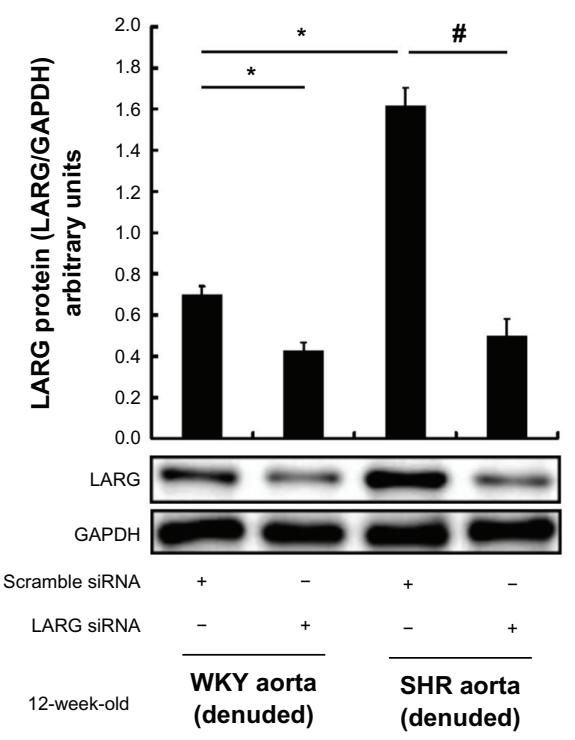

B1

WKY (si-scramble)

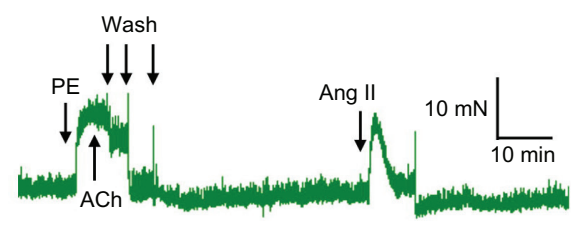

B3

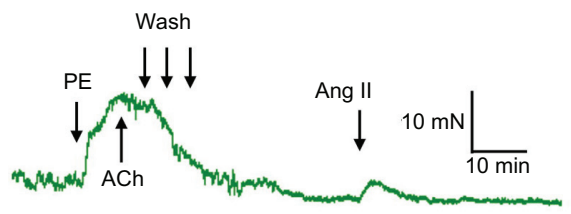

C

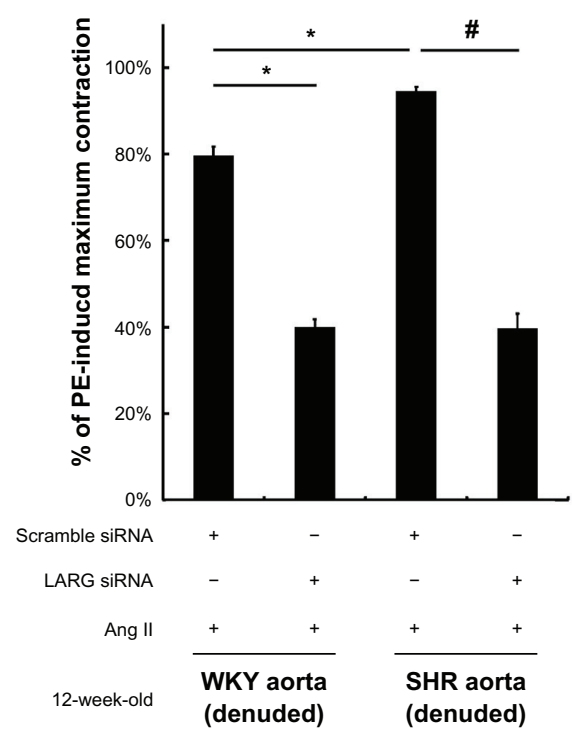

B2

SHR (si-scramble)

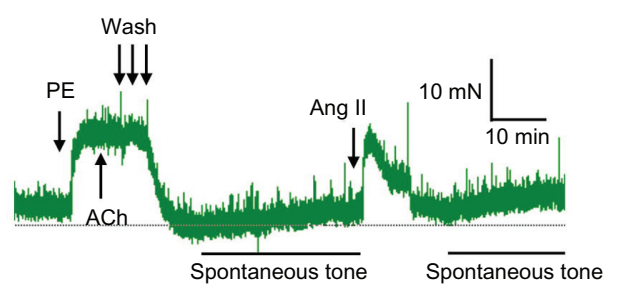

B4

SHR (si-LARG)

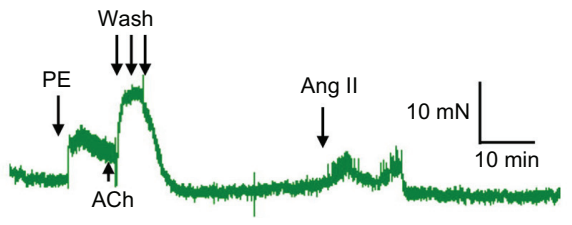

Figure 3 The effects of leukemia-associated Rho guanine nucleotide exchange factor (LARG) small interfering RNA (siRNA) on angiotensin II (Ang II)-induced aortic ring contraction in 12-week-old spontaneously hypertensive rats (SHRs) and Wistar-Kyoto (WKY) rats. Before contractile tension recording, LARG protein was extracted from endothelium-removal aortic segments pretreated with scrambled siRNA or LARG siRNA for 48 hours. The efficiency of LARG protein knockdown was evaluated by Western blot analysis, using glyceraldehyde-3-phosphate dehydrogenase (GAPDH) as an internal control. (A) A greater knockdown of LARG protein was observed in I2-week-old SHRs than in I2-week-old WKY rats; (BI-B4) typical recordings of contractile force present the effects of knockdown of LARG protein in the endotheliumdenuded aortic rings from SHRs and WKY rats - the level of spontaneous tone developed in SHR aortic rings treated with scrambled siRNA was $30.9 \% \pm 5.3 \%$ of phenylephrine (PE)-induced maximal contraction; (C) silencing of LARG expression attenuated more contractile force in SHRs than in WKY rats ( $58.0 \% \pm 2.6 \%$ versus $49.9 \% \pm 1.3 \%$, normalized to scrambled siRNA-treated control).

Notes: Data are presented as the means of four independent experiments plus or minus standard deviation; the scrambled siRNA-treated group was used as a negative control; $* P<0.01$ versus WKY rat negative control group; ${ }^{*} P<0.01$ versus SHR negative control group; the magnitude of contraction is expressed as a percentage of the maximal PE (I $\mu \mathrm{mol} / \mathrm{L})$-induced contraction.

Abbreviations: $\mathrm{ACh}$, acetylcholine; min, minute.

protein production was in parallel with increased levels of the corresponding mRNA in WKY rats. However, the baseline level of LARG protein of 12-week-old SHRs was approximately four times higher of that in WKY rats of the same age, although this level was not further increased after Ang II-stimulation. Third, the role of LARG in the development of spontaneous vascular tone and vasoconstriction in SHRs was confirmed by aortic ring contraction experiments. The knockdown of LARG protein expression attenuated contractile force in both SHRs and 
WKY rats, although the effect was more significant in SHRs than in WKY rats.

The age-dependent increase in the expression levels of three RhoGEF mRNAs in SHRs suggests there may be a growth and developmental transcriptional regulation. The higher the expression of RhoGEF, the more active the $\mathrm{Ca}^{2+}$ sensitization pathway RhoGEF/RhoA/Rho kinase/MLCP may be, which may lead to increased spontaneous tone and subsequently contribute to the hypertension genesis in mature SHRs. Compared with the two other RhoGEFs, only LARG mRNA and protein levels were significantly higher in 12-week-old SHRs than in 12-week-old WKY rats. Therefore, LARG may be a key regulator of the $\mathrm{Ca}^{2+}$ sensitization pathway during development of hypertension in 12-week-old SHRs. The mechanism of developmental differences in the expression of LARG in SHRs and WKY rats remains unclear and merits further study.

Some differences in the physiological and molecular properties of $\mathrm{Ca}^{2+}$ handling and $\mathrm{G}$ proteins between SHRs and WKY rats have been reported. ${ }^{31-33}$ These studies have shown that contribution of RhoA signaling to $\mathrm{Ca}^{2+}$ sensitivity in SHRs is greater than in WKY rats. Nevertheless, in contrast to protein activity, there is no difference in RhoA signaling-related molecule protein levels between mature SHRs and WKY rats. ${ }^{2}$ Data from the present study also show a higher baseline protein expression of the RhoA upstream LARG in 12-week-old SHRs than in age-matched WKY rats, suggesting that the activity of downstream RhoA signaling may be increased in 12-week-old SHRs.

Ying et $\mathrm{al}^{15}$ has reported that the LARG mRNA level was higher in 12-week-old stroke-prone SHRs than in agematched WKY rats; however, the authors do not mention comparisons of baseline and levels stimulated by $\mathrm{AT}_{1}$ receptor agonist between SHRs and WKY rats. The present authors' findings show that the pattern of LARG protein expression in 12-week-old WKY rats was different from that in age-matched SHRs. In 12-week-old WKY rats, both a lower baseline and a significant increase of LARG protein level following Ang II-stimulation were noted; however, the LARG protein level remained unchanged after Ang II-stimulation in 12-week-old SHRs. This suggests that the baseline level of LARG protein in SHRs was already expressed at a relatively high level and Ang II did not induce further increase in the expression level. Findings of the present study may be explained according to previous studies reporting that the plasma level of Ang II was not different between SHRs and WKY rats but that the Ang II receptor density in vascular wall was higher in SHRs than in WKY rats, ${ }^{14,34}$ which illustrates the higher LARG/RhoA/ Rho kinase/MYPT1 activity in SHRs.

Wirth et $\mathrm{al}^{7}$ reported that the aortic rings from LARGdeficient mice had diminished Ang II-induced contraction compared with the wild-type control. Likewise, data from the present study demonstrated that the specific LARGknockdown of SHR aortic rings abolished spontaneous tone and diminished contractile force in response to Ang II. Furthermore, the effect of LARG knockdown on diminishing contractile forces observed was greater in SHR aortic rings than in WKY rat aortic rings. These findings indicate that LARG contributes to maintenance of the basal tone in blood vessels and may be augmented in hypertension, which further supports the role of LARG in the regulation of hypertension development in SHRs. This hypothesis is also consistent with a previous study showing a greater decrease in blood pressure in SHRs than in WKY rats by RhoA/Rho kinase inhibition. ${ }^{3}$ One question to be answered is whether the increased expression of RhoGEF is an initiator or a consequence of hypertension. Ying et $\mathrm{a}^{15}$ have suggested that the increased expression of RhoGEF is only a consequence of hypertension. However, Wirth et $\mathrm{al}^{7}$ have shown that arteries in LARG knockout mice displayed reduced contractions in response to Ang II and failed to develop deoxycorticosterone acetate-induced hypertension. The findings of the present study are compatible with the results reported by Wirth et al, ${ }^{7}$ suggesting that increased expression of LARG could be an initiator rather than a consequence of hypertension.

Considering that Ang II has a half-life of about 30 seconds in circulation and possibly about 15 minutes in tissue, the transient increase of LARG mRNA at 30 minutes and disappearance at 3 hours in response to Ang II-stimulation was likely due to the short duration of action and decay of Ang II. Meanwhile, RNase may degrade LARG mRNA; the in vivo LARG mRNA expression may be related to continuous stimulation of intravascular Ang II - our in vitro observation was different from this. In addition, the protein will be present for 24-48 hours prior to proteolytic digestion after its synthesis. The authors' observations focused on the increase of gene expression following Ang II-stimulation and suggested that Ang II mediates the increased expression of RhoGEF. On the other hand, the cause of the decline in Ang II-stimulated RhoGEF mRNA expression after 6 hours in WKY rat groups of both ages was more likely an alteration during serum starvation of the cultured WKY rat VSMCs.

The results of a higher baseline expression of LARG in 12-week-old SHRs than in 5-week-old SHRs suggest 
that LARG may be an age-related causative factor for hypertension. If possible, a LARG-specific blocker (or inhibitor) could be used in 5-week-old SHRs to verify the development of hypertension when the rats reach 12 weeks of age. However, currently there is no such agent available to allow further investigation. On the other hand, considering that $\mathrm{AT}_{1}$ receptor density is high in $\mathrm{SHRs},{ }^{5}$ treatment with an $\mathrm{AT}_{1}$ antagonist for 5-week-old SHRs followed by evaluation of the inhibition of LARG expression when the rats reach 12 weeks of age can further confirm the role of AT both as $_{1}$ a LARG gene expression regulator and in the development of hypertension. In addition, the results can also be used to support the authors' hypothesis that $\mathrm{AT}_{1}$ activation over time is responsible for the increased LARG expression in adult SHRs. Found to be an important regulatory protein in many clinical disorders and diseases, including cancers and hypertension, LARG is an attractive target for future studies investigating therapeutics. . $^{735,36}$

\section{Conclusion}

In conclusion, the authors have shown different agerelated expression levels of RhoGEFs in SHRs and WKY rats; these include the different baseline and stimulated expression of RhoGEFs. In particular, significant changes of LARG expression and LARG knockdown reduced excessive contraction of aortic rings in SHRs. These findings support the authors' hypothesis that LARG may be one of the potent regulators of hypertension genesis in SHRs. The findings in this study also suggest that nanoparticle liposomes are effective for delivering siRNA across the cell membrane of aortic tissues. The siRNA delivery efficiencies enabled the authors both to evaluate the effects of RhoGEF knockdown on expression levels of mRNA and protein in the VSMCs of SHRs and to investigate consequent effects induced by Ang II-stimulation. Furthermore, the results indicate that the study model in combination with the use of synthetic siRNA can be suitable for more in-depth studies or as a potential therapeutic application for hypertension.

\section{Acknowledgments}

This study was supported by a grant (NSC98-2320-B002-035) from the National Science Council, Taiwan, and was supported in part by the Liver Disease Prevention \& Treatment Research Foundation, Taiwan.

\section{Disclosure}

The authors report no conflicts of interest in this work.

\section{References}

1. Guilluy C, Brégeon J, Toumaniantz G, et al. The Rho exchange factor Arhgef1 mediates the effects of angiotensin II on vascular tone and blood pressure. Nat Med. 2010;16(2):183-190.

2. Moriki N, Ito M, Seko T, et al. RhoA activation in vascular smooth muscle cells from stroke-prone spontaneously hypertensive rats. Hypertens Res. 2004;27(4):263-270.

3. Uehata M, Ishizaki T, Satoh H, et al. Calcium sensitization of smooth muscle mediated by a Rho-associated protein kinase in hypertension. Nature. 1997;389(6654):990-994.

4. Somlyo AP, Somlyo AV. Ca2+ sensitivity of smooth muscle and nonmuscle myosin II: modulated by $\mathrm{G}$ proteins, kinases, and myosin phosphatase. Physiol Rev. 2003;83(4):1325-1358.

5. Somlyo AP, Somlyo AV. Signal transduction by G-proteins, Rho-kinase and protein phosphatase to smooth muscle and non-muscle myosin II. $J$ Physiol. 2000;522 Pt 2:177-185.

6. Ying Z, Giachini FR, Tostes RC, Webb RC. Salicylates dilate blood vessels through inhibiting PYK2-mediated RhoA/Rho-kinase activation. Cardiovasc Res. 2009;83(1):155-162.

7. Wirth A, Benyó Z, Lukasova M, et al. G12-G13-LARG-mediated signaling in vascular smooth muscle is required for salt-induced hypertension. Nat Med. 2008;14(1):64-68.

8. Hilgers RH, Todd J Jr, Webb RC. Increased PDZ-RhoGEF/RhoA/Rho kinase signaling in small mesenteric arteries of angiotensin II-induced hypertensive rats. J Hypertens. 2007;25(8):1687-1697.

9. Rossman KL, Der CJ, Sondek J. GEF means go: turning on Rho GTPases with guanine nucleotide-exchange factors. Nat Rev Mol Cell Biol. 2005;6(2):167-180.

10. Bos JL, Rehmann H, Wittinghofer A. GEFs and GAPs: critical elements in the control of small G proteins. Cell. 2007;129(5):865-877.

11. Jaiswal M, Gremer L, Dvorsky R, et al. Mechanistic insights into specificity, activity, and regulatory elements of the regulator of G-protein signaling (RGS)-containing Rho-specific guanine nucleotide exchange factors (GEFs) p115, PDZ-RhoGEF (PRG), and leukemia-associated RhoGEF (LARG). J Biol Chem. 2011;286(20):18202-18212.

12. Ito K, Hirooka Y, Sakai K, et al. Rho/Rho-kinase pathway in brain stem contributes to blood pressure regulation via sympathetic nervous system: possible involvement in neural mechanisms of hypertension. Circ Res. 2003;92(12):1337-1343.

13. Yang RH, Jin HK, Wyss JM, Chen YF, Oparil S. Salt supplementation does not alter the pressor effect of blocking atrial natriuretic peptide in nucleus tractus solitarii. Hypertension. 1992;20(2):242-246.

14. Bunkenburg B, van Amelsvoort T, Rogg H, Wood JM. Receptor-mediated effects of angiotensin II on growth of vascular smooth muscle cells from spontaneously hypertensive rats. Hypertension. 1992;20(6): 746-754.

15. Ying Z, Jin L, Dorrance AM, Webb RC. Increaseed expression of mRNA for regulator of $\mathrm{G}$ protein signaling domain-containing Rho guanine nucleotide exchange factors in aorta from stroke-prone spontaneously hypertensive rats. Am J Hypertens. 2004;17(10):981-985.

16. Chiu W-C, Juang J-M, Chang S-N, et al. Angiotensin II regulates LARG/ RhoA/MYPT1 axis in rat vascular smooth muscle. Acta Pharmacol Sin. In press 2012.

17. Guo P, Coban O, Snead NM, et al. Engineering RNA for targeted siRNA delivery and medical application. Adv Drug Deliv Rev. 2010;62(6): 650-666.

18. Gao Y, Liu XL, Li XR. Research progress on siRNA delivery with nonviral carriers. Int J Nanomedicine. 2011;6:1017-1025.

19. Ross R. The smooth muscle cell: II. Growth of smooth muscle in culture and formation of elastic fibers. J Cell Biol. 1971;50(1):172-186.

20. Eguchi S, Hirata Y, Imai T, Kanno K, Marumo F. Phenotypic change of endothelin receptor subtype in cultured rat vascular smooth muscle cells. Endocrinology. 1994;134(1):222-228.

21. Eguchi S, Numaguchi K, Iwasaki H, et al. Calcium-dependent epidermal growth factor receptor transactivation mediates the angiotensin IIinduced mitogen-activated protein kinase activation in vascular smooth muscle cells. J Biol Chem. 1998;273(15):8890-8896. 
22. Yamakawa T, Tanaka S, Numaguchi K, et al. Involvement of Rho-kinase in angiotensin II-induced hypertrophy of rat vascular smooth muscle cells. Hypertension. 2000;35(1 Pt 2):313-318.

23. Alameh M, Jean M, DeJesus D, Buschmann MD, Merzouki A. Chitosanase-based method for RNA isolation from cells transfected with chitosan/siRNA nanocomplexes for real-time RT-PCR in gene silencing. Int J Nanomedicine. 2010;5:473-481.

24. Ying Z, Jin L, Palmer T, Webb RC. Angiotensin II up-regulates the leukemia-associated Rho guanine nucleotide exchange factor (RhoGEF), a regulator of $\mathrm{G}$ protein signaling domain-containing RhoGEF, in vascular smooth muscle cells. Mol Pharmacol. 2006;69(3):932-940.

25. Ying Z, Giachini FR, Tostes RC, Webb RC. PYK2/PDZ-RhoGEF links Ca2+ signaling to RhoA. Arterioscler, Thromb Vasc Biol. 2009;29(10): 1657-1663.

26. Lodi F, Cogolludo A, Duarte J, et al. Increased NADPH oxidase activity mediates spontaneous aortic tone in genetically hypertensive rats. Eur J Pharmacol. 2006;544(1-3):97-103.

27. Guilluy C, Rolli-Derkinderen M, Loufrani L, et al. Ste20-related kinase SLK phosphorylates Ser 188 of RhoA to induce vasodilation in response to angiotensin II type 2 receptor activation. Circ Res. 2008;102(10): $1265-1274$.

28. Sun D, Yan C, Jacobson A, Jiang H, Carroll MA, Huang A. Contribution of epoxyeicosatrienoic acids to flow-induced dilation in arteries of male ERalpha knockout mice: role of aromatase. Am J Physiol Regul Integr Comp Physiol. 2007;293(3):R1239-R1246.

29. Toumaniantz G, Ferland-McCollough D, Cario-Toumaniantz C, Pacaud $\mathrm{P}$, Loirand $\mathrm{G}$. The Rho protein exchange factor Vav3 regulates vascular smooth muscle cell proliferation and migration. Cardiovasc Res 2010;86(1):131-140.
30. Oh YK, Park TG. siRNA delivery systems for cancer treatment. $A d v$ Drug Deliv Rev. 2009;61(10):850-862.

31. Shimizu E, Ohyanagi M, Masutani M, Iwasaki T. A decrease in the amount and function of the stimulatory GTP-binding protein in the small resistance arteries of spontaneously hypertensive rats. Hypertens Res. 2002;25(5):743-749.

32. Asano M, Nomura Y. Calcium buffering of resting, voltage-dependent $\mathrm{Ca} 2+$ influx by sarcoplasmic reticulum in femoral arteries from spontaneously hypertensive rats at prehypertensive stage. Hypertens Res. 2001;24(3):271-282.

33. Asano M, Nomura Y. Ca2+ buffering function of the sarcoplasmic reticulum is increased in the carotid artery from spontaneously hypertensive rats. Hypertens Res. 2002;25(2):221-230.

34. Brunner HR, Chang P, Wallach R, Sealey JE, Laragh JH. Angiotensin II vascular receptors: their avidity in relationship to sodium balance, the autonomic nervous system, and hypertension. J Clin Invest. 1972;51(1): 58-67.

35. Schoner W. Salt abuse: the path to hypertension. Nat Med. 2008;14(1): 16-17.

36. Evelyn CR, Ferng T, Rojas RJ, Larsen MJ, Sondek J, Neubig RR. Highthroughput screening for small-molecule inhibitors of LARG-stimulated RhoA nucleotide binding via a novel fluorescence polarization assay. J Biomol Screen. 2009;14(2):161-172.
International Journal of Nanomedicine

\section{Publish your work in this journal}

The International Journal of Nanomedicine is an international, peerreviewed journal focusing on the application of nanotechnology in diagnostics, therapeutics, and drug delivery systems throughou the biomedical field. This journal is indexed on PubMed Central, MedLine, CAS, SciSearch $₫$, Current Contents $₫ /$ Clinical Medicine,

\section{Dovepress}

Journal Citation Reports/Science Edition, EMBase, Scopus and the Elsevier Bibliographic databases. The manuscript management system is completely online and includes a very quick and fair peer-review system, which is all easy to use. Visit http://www.dovepress.com/ testimonials.php to read real quotes from published authors. 